\title{
Identification of (-)-epigallocatechin (EGC) as a methylglyoxal (MGO)-trapping agent and thereby as an inhibitor of advanced glycation end product (AGE) formation
}

\author{
Eun Ryeol Shin ${ }^{1} \cdot$ Woong Jung ${ }^{2} \cdot$ Mi Kyoung Kim ${ }^{1} \cdot$ \\ Youhoon Chong ${ }^{1}$ (D)
}

Received: 3 June 2018/Accepted: 15 July 2018/Published online: 23 July 2018

(C) The Korean Society for Applied Biological Chemistry 2018

\begin{abstract}
Non-enzymatic glycosylation of proteins results in the formation of advanced glycation end products (AGEs). AGE modification of proteins and thereby damages to cells and tissues have been confirmed to contribute to the pathophysiology of aging and long-term complications of various age-related diseases. Anti-AGEs therapy has thus received significant attention, and several flavonoids including quercetin (1) and (-)-epigallocatechin gallate (EGCG) (2) have shown anti-AGEs activity through trapping and inactivating methylglyoxal (MGO), the crucial intermediate of AGEs formation. However, in the field of MGO-scavenging activity, (-)-epigallocatechin (EGC) (3), one of the key flavonoids in green tea, has received less attention compared with other flavonoids. In this study, we have shown strong MGO-scavenging activity of EGC (3), and EGC (3) was found to be equipotent to previously identified MGO-scavengers such as quercetin (1) and EGCG (2).
\end{abstract}

Keywords EGC · EGCG - Quercetin - MGO-scavenger · Advanced glycation end products $\cdot$ Inhibition

Youhoon Chong

chongy@konkuk.ac.kr

1 Department of Integrative Bioscience and Biotechnology, Bio/Molecular Informatics Center, Konkuk University, 120 Neungdong-ro, Gwangjin-gu, Seoul 05029, Korea

2 Department of Emergency Medicine, Kyung Hee University Hospital at Gangdong, Seoul 134-727, Korea

\section{Introduction}

Advanced glycation end products (AGEs) result from nonenzymatic glycosylation of macromolecules (proteins, phospholipids, and nucleic acids) upon exposure to glucose, fructose, and trioses. AGE modification of proteins results in protein dysfunction as well as damage in cells and tissues, and it has been confirmed that AGE-induced damage contributes to the pathophysiology of aging and long-term complications of diabetes [1], neurological diseases [2], atherosclerosis [3], and renal failure [4].

AGE formation is a complicated process, and many reactive intermediates are involved in this reaction. Among those, dicarbonyl intermediates such as glyoxal (GO), methylglyoxal (MGO), and 3-deoxyglucosone (3DG) have been reported as crucial precursors of AGEs because, due to the presence of reactive carbonyl groups, they can modify proteins to form AGEs of various chemical structures [5]. In particular, MGO has been given much attention because of its possible clinical significance in diabetes and its related complications [6].

Trapping and inactivating MGO have thus been recognized as the most viable approach to anti-AGEs therapy, and many MGO-scavengers [7] have been reported. However, side effects associated with these compounds impeded further development as antiglycative agents [8]. Accordingly, safety has become one of the most important requirements for MGO-scavengers and thereby anti-AGEs agents.

Natural compounds from plant sources, which are characterized by proven safety profiles along with broadspectrum bioactivities, have thus become the subject of investigation for development of safe MGO-scavengers [9-11]. Flavonoids have also been tested for MGO- 
Fig. 1 Structures of flavonoids investigated in this study<smiles>O=c1c(O)c(-c2ccc(O)c(O)c2)oc2cc(O)cc(O)c12</smiles>

Quercetin (Que) (1)

scavenging activity, and some of them including quercetin (1, Fig. 1) [12] and (-)-epigallocatechin-3-gallate (EGCG) (2, Fig. 1) [13] showed significant MGO-scavenging activity.

(-)-Epigallocatechin (EGC) (3, Fig. 1), another major flavonoid rich in green tea, also has various health-promoting effects [14, 15], but in the field of MGO-scavenging activity, EGC (3) has received less attention compared with EGCG (2). Therefore, in the course of our ongoing efforts to discover safe MGO-scavengers, it was of our interest to examine MGO-scavenging activity as well as anti-AGEs activity of EGC (3) in comparison with other flavonoids such as EGCG (2) and quercetin (1).<smiles>[R]C(=O)c1cc(O)c(O)c(O)c1</smiles>

\section{Materials and methods}

\section{Materials}

Flavonoids were purchased from Xi' an Le Sen Bio-technology (EGC and EGCG) (Xi' an City, China) and SigmaAldrich (quercetin) (St. Louis, MO. USA). MGO (5 mM in $100 \mathrm{mM}$ phosphate buffer) and 1,2-diaminobenzene (DB) were purchased from Sigma-Aldrich (St. Louis, MO. USA). BSA, dimethyl sulfoxide (DMSO), and phosphatebuffered saline (PBS) were obtained from RMBIO (Missoula, MT, USA), Merck Millipore (Burlington, MA, USA), and HyClone Laboratories (Logan, UT, USA),
Fig. 2 Trapping of MGO by (A) EGC (3), (B) EGCG (2), and (C) quercetin (1). MGO was incubated with the test flavonoids $(0.25,0.5,1.5$, and $2.5 \mathrm{mM}$ ) in $\mathrm{pH} 7.4$ phosphate buffer solutions at $37^{\circ} \mathrm{C}$ for 10 , $30,60,120$, and $240 \mathrm{~min}$. The remaining MGO was reacted with DB to give

methylquinoxaline which was quantified by HPLC. Each value represents the mean $\pm \mathrm{SD}$ $(n=3)$
(A)

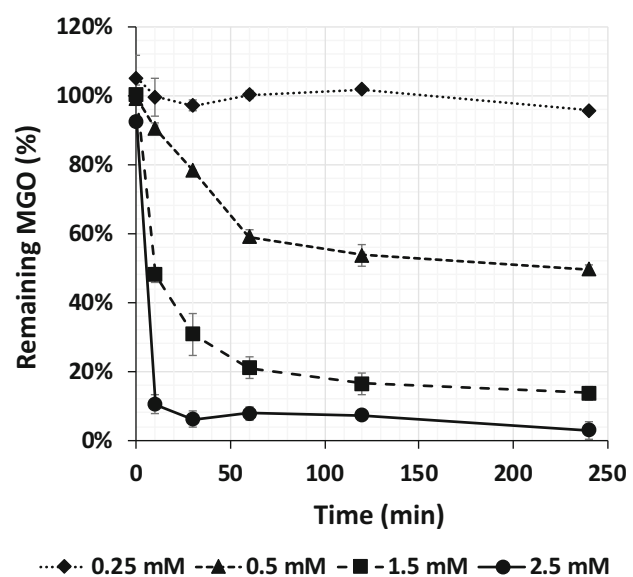

(B)

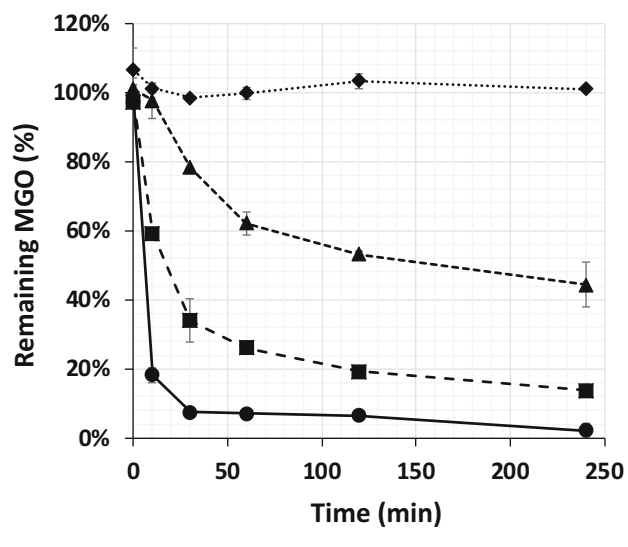

$0.25 \mathrm{mM}--0.5 \mathrm{mM}-\mathbf{a}-1.5 \mathrm{mM} \longrightarrow 2.5 \mathrm{mM}$

(C)

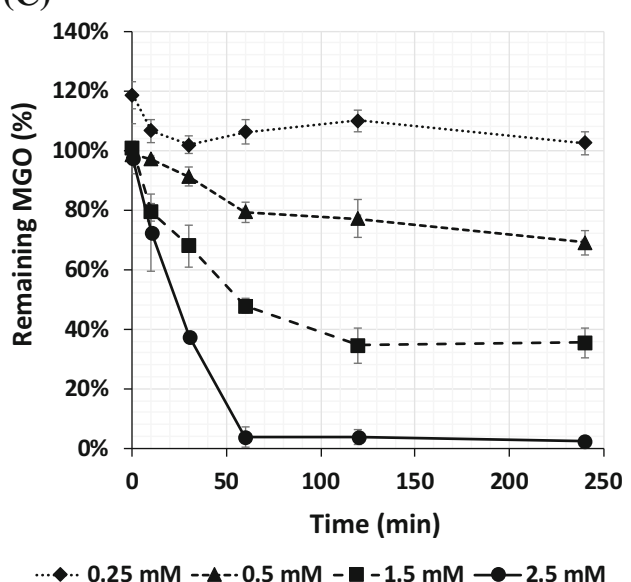


respectively. AGEs fluorescence ( $\lambda_{\mathrm{ex}} 340 \mathrm{~nm} ; \lambda_{\mathrm{em}} 420 \mathrm{~nm}$ ) was measured in a black plate 96 well (SPL Life Sciences, Gyeonggi-do, Korea) by using a CYTATION5 image reader (Biotek, Winooski, VT, USA).

\section{MGO-scavenging assay}

\section{Trapping of $M G O$}

MGO (5 mM) was incubated with four different concentrations $(0.25,0.5,1.5$, and $2.5 \mathrm{mM})$ of EGC (3), EGCG (2), or quercetin (1) in phosphate buffer (pH 7.4, $100 \mathrm{mM})$ at $37^{\circ} \mathrm{C}$. Shaking at $40 \mathrm{rpm}$ was continued for $0,10,30$, 60,120 , or $240 \mathrm{~min}$, and at each time point, reaction was stopped by addition of AcOH $(10 \mu \mathrm{L})$. DB (20 mM, 125 $\mu \mathrm{L})$ was added to the reaction mixture to derivatize the

(A)

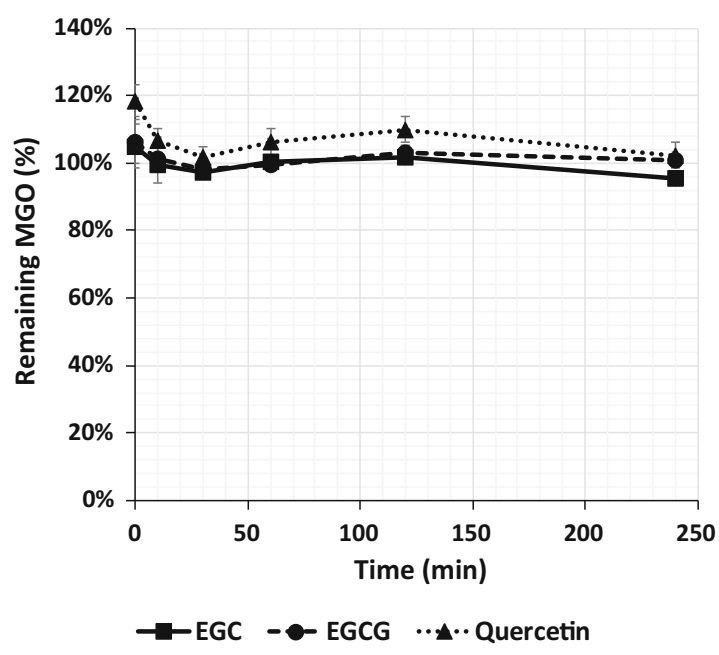

(C)

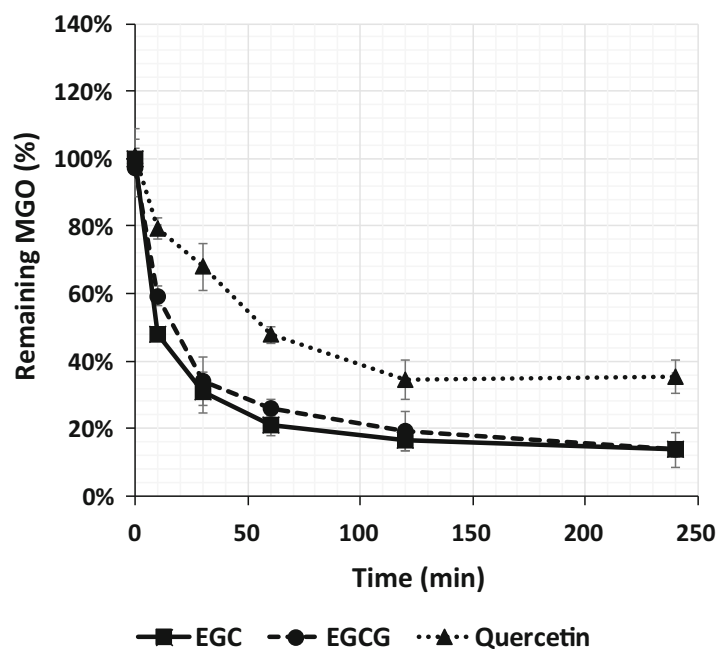

remaining MGO to methylquinoxaline. After keeping at room temperature for $30 \mathrm{~min}$, the reaction mixture was analyzed by HPLC to quantify methylquinoxaline, a surrogate of $\mathrm{MGO}$.

\section{HPLC analysis}

Methylquinoxaline, a surrogate of the remaining MGO, was analyzed by HPLC [UHPLC, Thermo-fisher, Waltham, MA, USA; Polaris C18-A column $(250 \mathrm{~mm} \times 4.6 \mathrm{~mm})$, Agilent Technologies, Santa Clara, CA, USA; flow rate = $1 \mathrm{~mL} / \mathrm{min}$; injection volume $=15 \mu \mathrm{L}]$. Mobile phase was composed of water containing $0.1 \% \mathrm{HCO}_{2} \mathrm{H}$ (phase A) and $\mathrm{MeOH}$ (phase $\mathrm{B}$ ), and the following linear gradient for elution was applied: 0-3 min, 5-50\% B; 3-16 min, 50-50\% B; 16-17 $\min , 50-90 \% \mathrm{~B} ; 17-22 \mathrm{~min}, 90-90 \% \mathrm{~B}$;

(B)

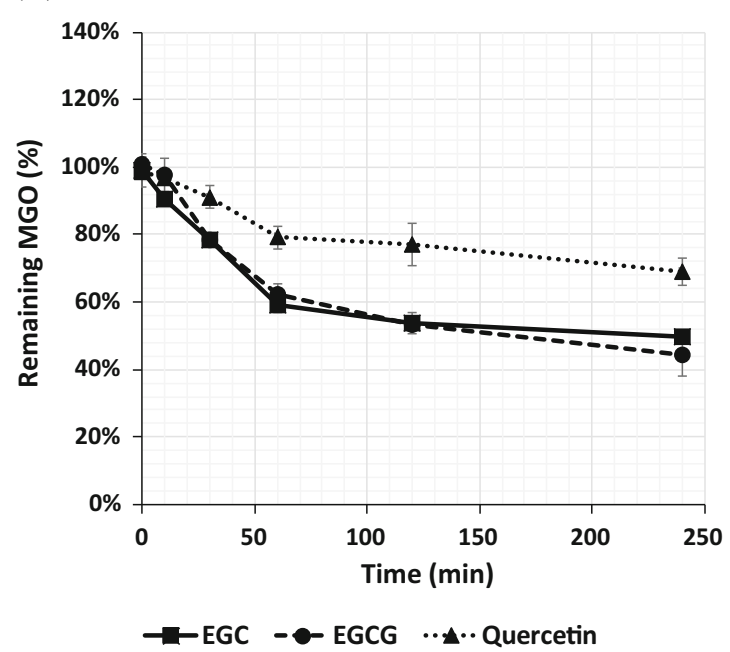

(D)

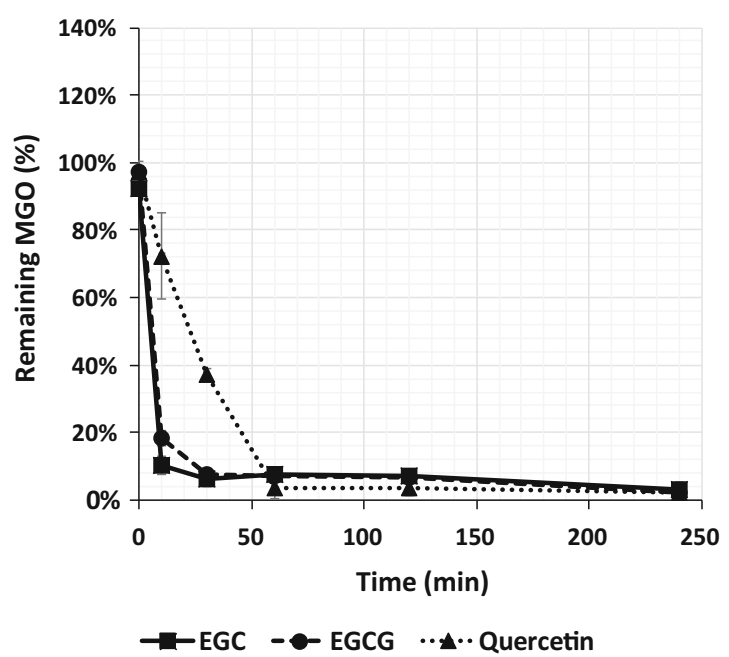

Fig. 3 Comparison of MGO-trapping activity of EGC (3), EGCG (2), and quercetin (1) in different concentrations [(A) $0.25 \mathrm{mM},(\mathbf{B}) 0.5 \mathrm{mM}$, (C) $1.5 \mathrm{mM}$, and (D) $2.5 \mathrm{mM}$. Each value represents the mean $\pm \mathrm{SD}(n=3)$ 
22-22.5 min, 90-5\% B. Methylquinoxaline eluted at $9.2 \min \left(\lambda_{\max }=315 \mathrm{~nm}\right)$, which was used to calculate the remaining MGO: remaining $\mathrm{MGO}(\%)=$ (amount of methylquinoxaline in the presence of the test compound/ amount of methylquinoxaline in control) $\times 100$.

\section{BSA-MGO assay}

BSA $(35 \mathrm{mg} / \mathrm{mL})$ and MGO $(5 \mathrm{mM})$ were incubated in $100 \mathrm{mM}$ phosphate buffer $\left(\mathrm{pH} \mathrm{7.4,37}{ }^{\circ} \mathrm{C}\right)$ for 7 days along with $0.25-2.5 \mathrm{mM}$ of the test flavonoids [EGC (3), EGCG (2), quercetin (1)]. Fluorescence of the control (no flavonoid added) and the test samples were measured $\left(\lambda_{\text {ex- }}\right.$ $\left.=340 \mathrm{~nm}, \lambda_{\mathrm{em}}=420 \mathrm{~nm}\right)$, which was used to calculate the \%-inhibition of AGEs formation by the flavonoids: [1(fluorescence from the test sample/fluorescence from the control)] $\times 100 \%$.

\section{Results and discussion}

\section{MGO-scavenging assay}

MGO-scavenging activity of EGC (3), EGCG (2), and quercetin (1) was determined by following previously published protocols [16]. The results summarized in Fig. 2 show that EGC (3) decreased MGO in a dose-dependent manner and, at $2.5 \mathrm{mM}$, no appreciable amount of MGO was detected after $10 \mathrm{~min}$ of incubation (Fig. 2A). EGCG (2) and quercetin (1) showed almost the same pattern in reducing MGO, and MGO levels dropped depending on the concentrations of the flavonoids as well as the time of incubation (Figs. 2B, 2C, respectively). In Fig. 3, the kinetics of MGO-scavenging activity of EGC (3), EGCG (2), and quercetin (1) was compared at different

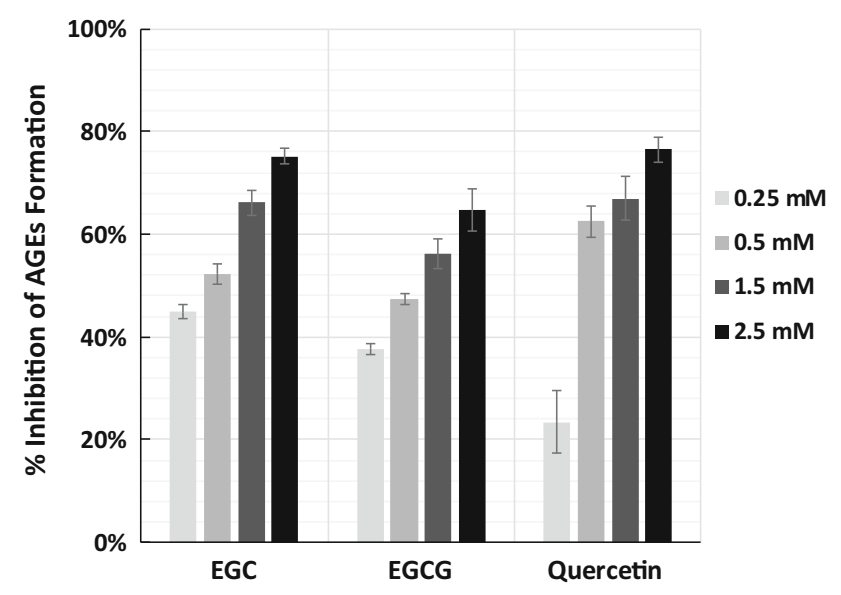

Fig. 4 Inhibitory effects of EGC (3), EGCG (2), and quercetin (1) on the AGE formation determined by BSA-MGO assay. Each value represents the mean $\pm \operatorname{SD}(n=3)$ concentrations $(0.25,0.5,1.5$, and $2.5 \mathrm{mM})$. In these plots, it is clearly shown that EGC (3) and EGCG (2) are equally potent in scavenging MGO, while quercetin (1) is less efficient than EGC (3) and EGCG (2).

\section{BSA (bovine serum albumin)-MGO assay}

Mechanistically, the non-enzymatic glycation of proteins, Maillard reaction, is composed of three stages [2], and the BSA-MGO assay is a specific method for investigation of inhibitors on middle stage of the glycation of protein. The BSA-MGO assay was performed by incubating BSA and MGO in the absence or presence of EGC, EGCG, or quercetin in phosphate buffer ( $\mathrm{pH} 7.4)$. Under these assay conditions, a reaction between BSA and MGO produces AGEs, which are easily detectable through fluorescence spectroscopy $\left(\lambda_{\mathrm{ex}}=340 \mathrm{~nm}, \lambda_{\mathrm{em}}=420 \mathrm{~nm}\right)$. The $\%$-inhibition of AGEs formation by the flavonoids is summarized in Fig. 4. As anticipated by the MGO-scavenging activity, the flavonoids [EGC (3), EGCG (2), and quercetin (1)] showed inhibitory activity against AGEs formation in a dose-dependent manner (Fig. 4). In the presence of EGC (3), the inhibition efficiency was found to increase with increasing concentration of EGC (3) from 0.25 to $2.5 \mathrm{mM}$. The maximum inhibition efficiency $77 \%$ was observed in the presence of $2.5 \mathrm{mM}$ of EGC (3) in the BSA-MGO assay system (Fig. 4).

In summary, AGE modification of proteins and thereby damages to cells and tissues have been confirmed to contribute to the pathophysiology of aging and long-term complications of various age-related diseases. Anti-AGEs therapy has thus received significant attention, and several flavonoids have shown anti-AGEs activity through trapping and inactivating $\mathrm{MGO}$, the crucial intermediate of AGEs formation. However, in the field of MGO-scavenging activity, EGC (3) has received less attention compared with other flavonoids. In this study, we have shown strong MGO-scavenging activity of EGC (3), and EGC (3) was equipotent to previously identified MGO-scavengers such as EGCG (2) and quercetin (1).

Acknowledgments This research was supported by the Basic Science Research Program through the National Research Foundation of Korea (NRF) funded by the Ministry of Science, ICT and future Planning (2015R1A2A1A10053461), as well as by a grant from the Priority Research Centers Program through the National Research Foundation of Korea (NRF) funded by the Ministry of Education, Science and Technology (2009-0093824).

\section{References}

1. Singh R, Barden A, Mori T, Beilin L (2001) Advanced glycation end-products: a review. Diabetologia 44:129-146 
2. Takeuchi M, Yamagishi S (2008) Possible involvement of advanced glycation end-products (AGEs) in the pathogenesis of Alzheimer's disease. Curr Pharm Des 14:973-978

3. Jandeleit-Dahm K, Cooper ME (2008) The role of AGEs in cardiovascular disease. Curr Pharm Des 14:979-986

4. Bohlender JM, Franke S, Stein G, Wolf G (2005) Advanced glycation end products and the kidney. Am J Physiol Renal Physiol 289:F645-F659

5. Beisswenger P, Howell S, Nelson R, Mauer M, Szwergold B (2003) $\alpha$-Oxoaldehyde metabolism and diabetic complications. Biochem Soc Trans 31:1358-1363

6. Wang H, Meng QH, Gordon JR, Khandwala H, Wu L (2007) Proinflammatory and proapoptotic effects of methylglyoxal on neutrophils from patients with type 2 diabetes mellitus. Clin Biochem 40:1232-1239

7. Thomas MC, Baynes JW, Thorpe SR, Cooper ME (2005) The role of AGEs and AGE inhibitors in diabetic cardiovascular disease. Curr Drug Targets 6:453-474

8. Nenna A, Nappi F, Avtaar Singh SS, Sutherland FW, Di Domenico F, Chello M, Spadaccio C (2015) Pharmacologic approaches against advanced glycation end products (AGEs) in diabetic cardiovascular disease. Res Cardiovasc Med 23:e26949

9. Liu H, Gu L (2012) Phlorotannins from Brown Algae (Fucus vesiculosus) inhibited the formation of advanced glycation endproducts by scavenging reactive carbonyls. J Agric Food Chem 60:1326-1334
10. Kumagai Y, Nakatani S, Onodera H, Nagatomo A, Nishida N, Matsuura Y, Kobata K, Wada M (2015) Anti-glycation effects of Pomegranate (Punica granatum L.) fruit extract and its components in vivo and in vitro. J Agric Food Chem 63:7760-7764

11. Zhang Y, Ma H, Liu W, Yuan T, Seeram NP (2015) New antiglycative compounds from Cumin (Cuminum cyminum) spice. J Agric Food Chem 63:10097-10102

12. Li X, Zheng T, Sang S, Lv L (2014) Quercetin inhibits advanced glycation end products formation by trapping methylglyoxal and glyoxal. J Agric Food Chem 62:12152-12158

13. Sang S, Shao X, Bai N, Lo C, Yang CS, Ho C (2007) Tea polyphenol (-)-epigallocatechin-3-gallate: a new trapping agent of reactive dicarbonyl species. Chem Res Toxicol 20:1862-1870

14. Vergote D, Cren-Olivé C, Chopin V, Toillon RA, Rolando C, Hondermarck H, Le Bourhis X (2002) (-)-Epigallocatechin (EGC) of green tea induces apoptosis of human breast cancer cells but not of their normal counterparts. Breast Cancer Res Treat 76:195-201

15. Yang GY, Liao J, Kim K, Yurkow EJ, Yang CS (1998) Inhibition of growth and induction of apoptosis in human cancer cell lines by tea polyphenols. Carcinogenesis 19:611-616

16. Shao X, Chen H, Zhu Y, Sedighi R, Ho C, Sang S (2014) Essential structural requirements and additive effects for flavonoids to scavenge methylglyoxal. J Agric Food Chem 62:3202-3210 